Published in final edited form as:

Nat Methods. 2019 November ; 16(11): 1176-1184. doi:10.1038/s41592-019-0583-8.

\title{
Machine learning-guided channelrhodopsin engineering enables minimally-invasive optogenetics
}

\author{
Claire N. Bedbrook ${ }^{1}$, Kevin K. Yang ${ }^{2, \dagger}$, J. Elliott Robinson ${ }^{1, \dagger}$, Elisha D. Mackey ${ }^{1}$, Viviana \\ Gradinaru ${ }^{1,{ }^{*}}$, Frances H. Arnold ${ }^{1,2,{ }^{*}}$ \\ ${ }^{1}$ Division of Biology and Biological Engineering; California Institute of Technology; Pasadena; \\ California; USA \\ ${ }^{2}$ Division of Chemistry and Chemical Engineering; California Institute of Technology; Pasadena; \\ California; USA
}

\section{Abstract}

\begin{abstract}
We engineered light-gated channelrhodopsins (ChRs) whose current strength and light sensitivity enable minimally-invasive neuronal circuit interrogation. Current $\mathrm{ChR}$ tools applied to the mammalian brain require intracranial surgery for transgene delivery and implantation of invasive fiber-optic cables to produce light-dependent activation of a small volume of tissue. To facilitate expansive optogenetics without the need for invasive implants, our engineering approach leverages the significant literature of $\mathrm{ChR}$ variants to train statistical models for the design of new, highperformance ChRs. With Gaussian Process models trained on a limited experimental set of 102 functionally characterized ChRs, we designed high-photocurrent ChRs with unprecedented light sensitivity; three of these, ChRger1-3, enable optogenetic activation of the nervous system via minimally-invasive systemic transgene delivery, not possible previously due to low per-cell transgene copy produced by systemic delivery. ChRger2 enables light-induced neuronal excitation without invasive intracranial surgery for virus delivery or fiber optic implantation, i.e. enables minimally-invasive optogenetics.
\end{abstract}

\section{Introduction}

Channelrhodopsins (ChRs) are light-gated ion channels found in photosynthetic algae. Transgenic expression of ChRs in the brain enables light-dependent neuronal activation ${ }^{1}$. These channels are widely applied as tools in neuroscience research ${ }^{2}$; however, functional

\footnotetext{
Users may view, print, copy, and download text and data-mine the content in such documents, for the purposes of academic research, subject always to the full Conditions of use:http://www.nature.com/authors/editorial_policies/license.html\#terms

*Corresponding: viviana@ caltech.edu (V.G.) \& fha@cheme.caltech.edu (F.H.A).

†K.K.Y. \& J.E.R. contributed equally to this work. Author Contributions

C.N.B., K.K.Y., V.G., and F.H.A. conceptualized the project. C.N.B. coordinated all experiments and data analysis. C.N.B. and K.K.Y. built machine-learning models. C.N.B. performed construct design and cloning. C.N.B. and E.D.M. performed AAV production. E.D.M. prepared cultured neurons. C.N.B and J.E.R. conducted electrophysiology. C.N.B. and J.E.R. performed injections. J.E.R. performed fiber cannula implants and behavioral experiments. C.N.B. performed all data analysis. C.N.B. wrote the manuscript with input and editing from all authors. V.G. supervised optogenetics/electrophysiology, and F.H.A. supervised the protein engineering. Competing interests A provisional patent application (CIT File No.: CIT-8092-P) has been filed by Caltech based on these results. C.N.B., K.K.Y., V.G., and F.H.A. are inventors on this provisional patent.
} 
limitations of available ChRs prohibit a number of optogenetic applications. These channels have broad activation spectra in the visible range and require high-intensity light for activation $\left[\sim 1 \mathrm{~mW} \mathrm{~mm}^{-2}\right]$. ChRs are naturally low-conductance channels requiring approximately $10^{5}-10^{6}$ functional ChRs expressed in the plasma-membrane of a neuron to produce sufficient light-dependent depolarization to induce neuronal activation ${ }^{3}$. When applied to the mouse brain, ChRs require $\sim 1-15 \mathrm{~mW}$ light delivered $\sim 100 \mu \mathrm{m}$ from the target cell population to reliably activate action potentials ${ }^{4-6}$. This confines light-dependent activation to a small volume of brain tissue $\left[\sim 1 \mathrm{~mm}^{3}\right]^{7}$. Enabling optogenetics for large brain volumes without the need to implant invasive optical fibers for light delivery would be highly desirable for neuroscience applications.

Engineering ChRs to overcome limits in conductance and light sensitivity and extend the reach of optogenetic experiments requires overcoming three major challenges. First, rhodopsins are trans-membrane proteins that are inherently difficult to engineer because the sequence and structural determinants of membrane protein expression and plasmamembrane localization are highly constrained and poorly understood ${ }^{8,9}$. Second, because properties of interest for neuroscience applications are assayed using low-throughput techniques, such as patch-clamp electrophysiology, engineering by directed evolution is not feasible ${ }^{10}$. And third, in vivo applications require retention and optimization of multiple properties, for example, localization in mammalian cells while simultaneously tuning kinetics, photocurrents, and spectral properties ${ }^{6}$.

Diverse ChRs have been published, including variants discovered from nature ${ }^{11,12}$, variants engineered through recombination ${ }^{9,13}$ and mutagenesis ${ }^{14,15}$, as well as variants resulting from rational design ${ }^{16}$. Studies of these coupled with structural information ${ }^{17}$ and molecular dynamic simulations ${ }^{18}$ have established some understanding of the mechanics and sequence features important for specific ChR properties ${ }^{1,16}$. Despite this, it is still not possible to predict functional properties of new $\mathrm{ChR}$ sequences.

Our approach has been to leverage the significant literature of ChRs to train statistical models that enable design of highly-functional ChRs. These models take as their input the sequence and structural information for a given $\mathrm{ChR}$ variant and then predict its functional properties. The models use training data to learn how sequence and structural elements map to ChR function. Once known, that mapping can be used to predict the behavior of untested ChR variants.

We trained models in this manner and found that they accurately predict the properties of untested ChR sequences. We used these models to engineer 30 'designer' $\mathrm{ChR}$ variants with specific combinations of desired properties, a number of which have unprecedented photocurrent strength and light sensitivity. We characterized these low-light sensitive, highphotocurrent $\mathrm{ChRs}$ for applications in the mammalian brain and demonstrate their potential for minimally-invasive activation of populations of neurons in the brain enabled by systemic transgene delivery with the engineered adeno-associated virus (AAV), rAAV-PHP.eB ${ }^{19}$. This work demonstrates how a machine learning-guided approach can enable engineering of proteins that have been challenging to engineer. 


\section{Results}

\section{Functional characterization of $\mathrm{ChR}$ variants for machine learning}

In previous work, we explored structure-guided recombination ${ }^{20,21}$ of three highlyfunctional ChR parents [CsChrimsonR $(\mathrm{CsChrimR})^{11}, \mathrm{C} 1 \mathrm{C} 2^{17}$, and CheRiff ${ }^{22}$ ] by designing two 10-block recombination libraries with a theoretical size of $\sim 120,000$ (i.e. $2 \times 3^{10}$ ) chimeric variants ${ }^{9}$. These recombination libraries are a rich source of functionally diverse sequences ${ }^{9}$. Previously, we generated $102 \mathrm{ChR}$ variants selected from these recombination libraries ${ }^{8,9}, 76$ of these were previously characterized for photocurrent properties (with patch-clamp electrophysiology) and 26 we characterized here. Together, these $102 \mathrm{ChR}$ recombination variants provide the primary dataset used for model training. We supplemented this dataset with data from other published sources including $19 \mathrm{ChR}$ variants from nature, 14 single-mutant $\mathrm{ChR}$ variants, and 28 recombination variants from other libraries (Dataset 1). As the data produced by other labs were not collected under the same experimental conditions as data collected in our hands, they cannot be used for comparison for absolute $\mathrm{ChR}$ properties (i.e. photocurrent strength); however, these data do provide useful binary information on whether a sequence variant is functional or not. Thus, we used published data from other sources when training binary classification models for $\mathrm{ChR}$ function.

Because our goal was to optimize photocurrent strength, wavelength sensitivity, and offkinetics, we used these measured properties to train machine-learning models (Figure 1a). Enhancing ChR photocurrent strength would enable reliable neuronal activation even under low-light conditions. Different off-rates can be useful for specific applications: fast offkinetics enable high-frequency optical stimulation ${ }^{23}$, slow off-kinetics is correlated with increased light sensitivity $3,14,15$, and very slow off-kinetics can be used for constant depolarization (step-function opsins [SFOs] ${ }^{14}$ ). In addition to opsin functional properties, it is also necessary to optimize or maintain plasma-membrane localization, a prerequisite for ChR function ${ }^{8}$.

\section{Training Gaussian process (GP) classification and regression models}

Using the $\mathrm{ChR}$ sequence/structure and functional data as inputs, we trained Gaussian process (GP) classification and regression models (Figure 1). GP models successfully predicted thermostability, substrate binding affinity, and kinetics for several soluble enzymes ${ }^{24}$, and, more recently, ChR membrane localization ${ }^{8}$. For a detailed description of the GP model architecture used for protein engineering see refs 8, 24. Briefly, these models infer predictive values for new sequences from training examples by assuming that similar inputs (ChR sequence variants) will have similar outputs (photocurrent properties). To quantify the relatedness of inputs (ChR sequence variants), we compared both sequence and structure. ChR sequence information is encoded in the amino acid sequence. For structural comparisons, we convert the 3D crystal-structural information into a 'contact map' that is convenient for modeling. Two residues are considered to be in contact and potentially important for structural and functional integrity if they have any non-hydrogen atoms within $4.5 \AA$ in the $\mathrm{C} 1 \mathrm{C} 2$ crystal structure (3UG9.pdb) ${ }^{17}$. We defined the sequence and structural 
similarity between two variants by aligning them and counting the number of positions and contacts at which they are identical ${ }^{24}$.

We trained a binary classification model to predict if a ChR sequence will be functional using all 102 training sequences from the recombination library (Dataset 2) as well as data from 61 variants published by others (Dataset 1). We then used this trained classification model to predict whether uncharacterized $\mathrm{ChR}$ sequence variants were functional (Figure 1b). To test prediction accuracy, we performed 20 -fold cross validation on the training data set and achieved an area under the receiver operator curve (AUC) of 0.78 , indicating good predictive power (Supplemental Table 1).

Next, we trained three regression models, one for each of the ChR photocurrent properties of interest: photocurrent strength, wavelength sensitivity of photocurrents, and off-kinetics (Figure 1c). Once trained, these models were used to predict photocurrent properties of new, untested ChRs sequence variants. To test prediction accuracy, we performed 20-fold cross validation on the training dataset and observed high correlation between predicted and measured properties for all models (Pearson correlation [R] between 0.77-0.9; Supplemental Table 1-2). Models built using contact maps from either the ChR2 crystal structure ${ }^{25}$ or C1Chrimson crystal structure ${ }^{26}$ perform as well as models built with a contact map from the C1C2 structure ${ }^{17}$ (Supplemental Table 3, Supplemental Figure 1c,d) even though these maps share only $82 \%$ and $89 \%$ of their contacts with the C1C2 map, respectively (Supplemental Figure 1a,b).

\section{Selection of designer ChRs using trained models}

To select 'designer' ChRs (i.e. ChRs predicted to have a useful combination of properties), we used a tiered approach (Figure 1d). First, we eliminated all ChR sequences predicted to not localize to the plasma membrane or predicted to be non-functional. To do this, we used classification models of $\mathrm{ChR}$ localization ${ }^{8}$ and function to predict the probability of localization and function for each ChR sequence in the 120,000-variant recombination library. Not surprisingly, most ChR variants were predicted to not localize and not function. To focus on ChR variants predicted to localize and function, we set a threshold for the product of the predicted probabilities of localization and function (Figure 1b); any $\mathrm{ChR}$ sequence above that threshold would be considered for the next tier of the process. We selected a conservative threshold of 0.4 .

The training data made clear that the higher the mutation distance from one of the three parents, the less likely it was that a sequence would be functional; however, we expect that more diverse sequences would also have the more diverse functional properties. To explore diverse sequences predicted to function, we selected $22 \mathrm{ChR}$ variants that passed the 0.4 threshold and were multi-block-swap sequences containing on average 70 mutations from the closest parent. These 22 sequences were synthesized, expressed in HEK cells, and their photocurrent properties were measured with patch-clamp electrophysiology. 59\% of the tested sequences were functional (Figure 1e), compared to $38 \%$ of the multi-block swap sequences randomly selected (i.e., not selected by the model) and having comparable average mutation level. This validates the classification model's ability to make useful predictions about novel functional sequences, even for sequences that are very distant from 
those previously tested. We updated the models by including data from these 22 sequences for future rounds of predictions.

From the 120,000-variant recombination library, 1,161 chimeric sequence variants passed the conservative 0.4 predicted localization and function threshold (Figure 1). For the second tier of the selection process, we used the three regression models trained on all functional variants collected up to this point to predict the photocurrent strength, wavelength sensitivity of photocurrents, and off-kinetics for each of these 1,161 ChR sequence variants (Dataset 3). We selected 28 designer ChRs predicted to be highly functional with different combinations of properties including those predicted to have the highest photocurrent strength, most redshifted or blue-shifted activation wavelengths, and off-kinetics from very fast to very slow (Supplemental Figure 2-3).

Genes encoding the 28 selected designer ChR variants were synthesized, expressed in HEK cells, and characterized for their photocurrent properties with patch-clamp electrophysiology. All 28 selected designer ChRs were functional: $100 \%$ of variants selected using the updated classification model above the 0.4 threshold both localize and function. For each of the designer $\mathrm{ChR}$ variants, the measured photocurrent properties correlated well with the model predictions ( $\mathrm{R}>0.9$ for all models) (Figure 1f, Supplemental Table 1). This outstanding performance on a novel set of sequences demonstrates the power of this datadriven predictive method for engineering designer ChRs. As a negative control, we selected two ChR variant sequences from the recombination library that the model predicted would be non-functional (ChR_29_10 and ChR_30_10). These sequences resulted from a singleblock swap from two of the most highly functional $\mathrm{ChR}$ recombination variants tested. As predicted, these sequences were non-functional (Figure 2b), which shows that ChR functionality can be attenuated by incorporating even minimal diversity at certain positions.

\section{Sequence and structural determinants of ChR functional properties}

We used L1-regularized linear regression models to identify a limited set of residues and structural contacts that strongly influence ChR photocurrent strength, spectral properties, and off-kinetics (Supplemental Figure 4a). We can assess the relative importance of each of these sequence and structural features by weighting their contributions using L2-regularized linear regression (Dataset 4 and Supplemental Figure 4). For each functional property, we identified a set of important residues and contacts and their respective weights. A specific residue or contact at a given position is weighted as likely to lead to, e.g., low (negative weight) or high (positive weight) photocurrents. A number of residues and contacts most important for tuning spectral properties are proximal to the retinal-binding pocket, including the blue-shifting contact between A206 and F269 and the blue-shifting contact between F265 and I267 that are conserved in the blue-shifted parents C1C2 and CheRiff while the red-shifting contact between F201 and Y217 originates from the red-shifted CsChrimR parent (Supplemental Figure 4). The most heavily weighted contact contributing to offkinetics includes the reside D195 (i.e., D156 according to ChR2 numbering) (Supplemental Figure 4), a residue that is part of the DC-gate ${ }^{1}$. Mutation of either the aspartic acid or cysteine within the DC-gate has been shown to decrease off-kinetic speed ${ }^{14,27}$. While the cysteine in the DC-gate is conserved in all three ChR parents, the aspartic acid at position 
195 is only conserved in CheRiff and C1C2 but not in CsChrimR, which has a cysteine at that position. Interestingly, D195 is also part of a contact with L192 that contributes strongly to photocurrent strength (Supplemental Figure 4). A number of contacts proximal to retinal contribute strongly to photocurrent strength. For example, the most heavily weighted contact includes A295 (from CsChrimR), which is adjacent to the conserved lysine residue that covalently links retinal (Supplemental Figure 4). This position is a serine in both C1C2 and CheRiff.

\section{Machine-guided search identifies ChRs with a range of useful functional properties}

We assessed photocurrent amplitude, wavelength sensitivity, and off-kinetics of the designer ChRs and the three parental ChRs (Figure 2). In addition to the 28 regression modelpredicted ChRs, we also assessed the two top-performing ChRs from the classification models' predictions (ChR_9_4 and ChR_25_9), for a total of 30 highly-functional modelpredicted ChRs as well as the two negative control ChRs (ChR_29_10, ChR_30_10). Of the 30 model-predicted ChRs, we found 12 variants with $\geq 2$-times higher blue-light activated photocurrents than the top-performing parent (CsChrimR) (Figure 2b). Three variants exhibit $\geq 1.7$-times higher green-light activated photocurrents than CsChrimR. Eight variants have larger red-light activated photocurrents when compared with the blue-light activated parents (CheRiff and $\mathrm{C} 1 \mathrm{C} 2$ ), though none out-perform CsChrimR. Both $\mathrm{ChR}$ variants predicted to be non-functional by the models produce $<30 \mathrm{pA}$ currents.

Designer ChRs' off-kinetics span three orders of magnitude ( $\tau_{\text {off }}=10 \mathrm{~ms}->10 \mathrm{~s}$ ) (Figure 2c). This range is quite remarkable given that all designer $\mathrm{ChRs}$ are built from sequence blocks of three parents that have similar off-kinetics ( $\tau_{\text {off }}=30-50 \mathrm{~ms}$ ). We found that 5 designer ChRs have faster off-kinetics than the fastest parent, while 16 have $>5$-times slower off-kinetics. The two fastest variants, ChR_3_10 and ChR_21_10 exhibit $\tau_{\text {off }}=13 \pm 0.9 \mathrm{~ms}$ and $12 \pm 0.4 \mathrm{~ms}$, respectively (mean \pm SEM). Four ChRs have particularly slow off-kinetics with $\tau_{\text {off }}>1 \mathrm{~s}$, including ChR_15_10, ChR_6_10, and ChR_13_10 ( $\tau_{\text {off }}=4.3 \pm 0.1 \mathrm{~s}, 8.0 \pm 0.5$ s, and $17 \pm 7 \mathrm{~s}$, respectively). Two ChRs with very large photocurrents, ChR_25_9 and ChR_11_10, exhibit $\tau_{\text {off }}=220 \pm 10 \mathrm{~ms}$ and $330 \pm 30 \mathrm{~ms}$, respectively.

Three designer ChRs exhibit interesting spectral properties (Figure 2e, Supplemental Figure 5). ChR_28_10's red-shifted spectrum matches that of CsChrimR, demonstrating that incorporating sequence elements from blue-shifted ChRs into CsChrimR can still generate a red-shifted activation spectrum. Two designer ChRs exhibit novel spectral properties: ChR_11_10 has a broad activation spectrum relative to the parental spectra, with similar steady-state current strength from 400-546 nm light and strong currents (700 $\pm 100 \mathrm{pA})$ when activated with $567 \mathrm{~nm}$ light. ChR_25_9, on the other hand, exhibits a narrow activation spectrum relative to the parental spectra, with a peak at $481 \mathrm{~nm}$ light.

We assessed the light sensitivity of select designer ChRs. Compared with CsChrimR, CheRiff, and $\mathrm{C} 1 \mathrm{C} 2$, the designer ChRs have $\geq 9$-times larger currents at the lowest intensity of light tested $\left(10^{-1} \mathrm{~mW} \mathrm{~mm}^{-2}\right)$, larger currents at all intensities of light tested, and minimal decrease in photocurrent magnitude over the range of intensities tested $\left(10^{-1}-10^{1} \mathrm{~mW} \mathrm{~mm}\right.$ -2 ), suggesting that photocurrents were saturated at these intensities and would only attenuate at much lower light intensities (Figure 2d). These select designer ChRs are 
expressed at levels similar to the $\mathrm{CsChrimR}$ parent (the highest expressing parent) indicating that the improved photocurrent strength of these ChRs is not solely due to improved expression (Supplemental Figure 6-7).

We compared three of the top designer ChRs (ChR_9_4, ChR_25_9, and ChR_11_10) with ChR2(H134R) ${ }^{6,28}$, an enhanced photocurrent single mutant of ChR2 commonly used for in vivo optogenetics, and CoChR (from Chloromonas oogama) ${ }^{11}$, reported to be one of the highest conducting ChRs activated by blue light ${ }^{11}$. The selected designer ChRs produce 36x larger photocurrents than ChR2(H134R) when exposed to high-intensity $\left(2.2 \mathrm{~mW} \mathrm{~mm}^{-2}\right.$ ) $481 \mathrm{~nm}$ light and 10-18x larger photocurrents than ChR2(H134R) when exposed to lowintensity $\left(6.5 \times 10^{-2} \mathrm{~mW} \mathrm{~mm}^{-2}\right) 481 \mathrm{~nm}$ light (Supplemental Figure 8f,g). Although CoChR produced peak currents of similar magnitude to the designer $\mathrm{ChRs}$, CoChR decays to a much lower steady-state level (Supplemental Figure 8d,e) with the designer ChRs producing 2-3x larger steady-state photocurrents than CoChR when exposed to high-intensity light and $3-4 x$ larger steady-state photocurrents than CoChR when exposed to low-intensity light (Supplemental Figure 8f,g; Supplemental Table 4). The increased low-light sensitivity of these select designer ChRs is likely due in part to their relatively slow off-kinetics leading to the increased accumulation of the open state under low-light conditions ${ }^{14}$.

\section{Validation of designer ChRs for neuroscience applications}

For further validation we selected three of the top high-conductance ChRs, ChR_9_4, ChR_25_9, and ChR_11_10, and renamed them ChRger1, ChRger2, and ChRger3,

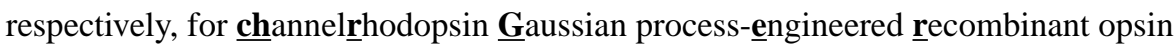
(Supplemental Figure 9). When expressed in cultured neurons, the ChRgers display robust membrane localization and expression throughout the neuron soma and neurites (Figure 3b). The ChRgers outperform both CoChR and ChR2(H134R) in photocurrent strength with lowintensity light in neuronal cultures (Figure 3c). The designer ChRgers require 1-2 orders of magnitude lower light intensity than CoChR and ChR2(H134R) for neuronal activation (Figure 3d, Supplemental Figure 8h).

Next, we performed direct intracranial injections into the mouse prefrontal cortex (PFC) of rAAV-PHP.eB packaging either ChRger1-3, or ChR2(H134R) under the hSyn promoter (Supplemental Table 5). After 3-5 weeks of expression, we measured light sensitivity in ChR-expressing neurons in acute brain slices. We observed greater light sensitivity for the ChRgers compared with ChR2(H134R) (Figure 3g,h). The ChRgers exhibit >200 pA photocurrent at $10^{-3} \mathrm{~mW} \mathrm{~mm}^{-2}$ while at the equivalent irradiance $\mathrm{ChR} 2$ (H134R) exhibits undetectable photocurrents. The ChRgers reach $>1000 \mathrm{pA}$ photocurrents with $\sim 10^{-2} \mathrm{~mW}$ $\mathrm{mm}^{-2}$ light, a four-fold improvement over ChR2(H134R)'s irradiance-matched photocurrents (Figure 3g). Our characterization of ChR2(H134R)'s light sensitivity and photocurrent strength is consistent with previous results from other labs ${ }^{6,22}$.

\section{Designer ChRs and systemic AAVs enable minimally-invasive optogenetic excitation}

We investigated whether these light-sensitive, high-photocurrent ChRs could provide optogenetic activation coupled with minimally-invasive gene delivery. Previous reports of 'non-invasive optogenetics' relied on invasive intracranial virus delivery, which results in 
many copies of virus per cell and thus very high expression levels of the injected construct $^{29}$. Recently, we described the AAV capsid rAAV-PHP.eB ${ }^{19}$ that produces broad transduction throughout the central nervous system with a single minimally-invasive intravenous injection in the adult mouse ${ }^{30,31}$. Systemic delivery of rAAV-PHP.eB results in brain-wide transgene delivery without invasive intracranial injections ${ }^{19,30,31}$. Use of rAAVPHP.eB for optogenetic applications has been limited, however, by the low multiplicity of infection with systemically delivered viral vectors resulting in insufficient opsin expression and light-evoked currents to control neuronal firing with commonly-used channels (e.g. ChR2).

We hypothesized that the ChRgers could overcome this limitation and allow large-volume optogenetic excitation following systemic transgene delivery. We systemically delivered rAAV-PHP.eB packaging either ChRger1, ChRger2, CoChR, or ChR2(H134R) under the hSyn promoter and observed broad expression throughout the brain (Figure 3i). We measured the fraction of opsin-expressing cells with sufficient opsin-mediated currents for light-induced firing (Figure 3j). Only 4\% of ChR2(H134R)-expressing neurons produced light-induced firing, while 77\% of CoChR-expressing neurons, $89 \%$ of ChRger1-expressing neurons, and $100 \%$ of ChRger2- or ChRger3-expressing neurons produced light-induced activity. With systemic delivery, we observed superior light sensitivity of ChRgers compared with $\mathrm{CoChR}$ in both photocurrent strength (Figure 3k) and spike fidelity (Figure 31).

ChRger2-expressing neurons exhibit healthy membrane properties similar to CoChR- or ChR2(H134R)-expressing neurons both in culture and in slice (Supplemental Figure 10; Supplemental Table 6). These results demonstrate the need for light-sensitive and highphotocurrent opsins for applications where systemic delivery is desired.

We systemically delivered rAAV-PHP.eB packaging ChRger1-3 under the CaMKIIa promoter. With systemic delivery of ChRger2, we observed photocurrent strength similar to results observed after direct injection into the PFC (Figure 3g). When expressed in pyramidal neurons in the cortex, ChRger2 and ChRger3 enabled robust optically-induced firing at rates between $2-10 \mathrm{~Hz}$, although spike fidelity was reduced at higher frequency stimulation (Figure $3 \mathrm{~m}, \mathrm{n}$ ). ChRger2 performed best with higher frequency stimulation while ChRger1 performed worst. CoChR has better spike fidelity than the ChRgers at higher frequency stimulation $(20-40 \mathrm{~Hz})$ (Figure $3 \mathrm{~m}$ ).

We next evaluated the optogenetic efficiency of ChRger2 after systemic delivery using optogenetic intracranial self-stimulation (oICSS) of dopaminergic neurons of the ventral tegmental area $(\mathrm{VTA})^{32}$. We systemically delivered rAAV-PHP.eB packaging a doublefloxed inverted open reading frame (DIO) containing either ChRger2 or ChR2(H134R) into Dat-Cre mice (Figure 4a and Supplemental Table 5). Three weeks after systemic delivery and stereotaxic implantation of fiber-optic cannulas above the VTA, mice were placed in an operant box and were conditioned to trigger a burst of $447 \mathrm{~nm}$ laser stimulation via nose poke. Animals expressing ChRger2 displayed robust optogenetic self-stimulation in a frequency-dependent and laser power-dependent manner. Higher frequencies (up to $20 \mathrm{~Hz}$ ) and higher light power (up to $10 \mathrm{~mW}$ ) promoted greater maximum operant response rates (Figure 4a). Conversely, laser stimulation failed to reinforce operant responding in ChR2(H134R)-expressing animals (Figure 4a); these results were consistent with results in 
acute slice where the light-induced currents of ChR2(H134R) are too weak at the low copy number produced by systemic delivery for robust neuronal activation.

In order to determine if ChRger2 would enable both minimally-invasive transgene delivery and minimally-invasive optical excitation, we assayed directional control of locomotion in freely moving animals by optogenetic stimulation of the right secondary motor cortex $(\mathrm{M} 2)^{33}$. In this assay, unilateral stimulation of M2 disrupts motor function in the contralateral lower extremities, causing mice to turn away from the stimulation side. We systemically administered rAAV-PHP.eB packaging either ChRger2 or ChR2(H134R) under a CaMKIIa promoter for transgene expression in excitatory pyramidal neurons in the cortex (Figure 4b, and Supplemental Table 5). We observed broad expression throughout the cortex for both ChRger2 and ChR2(H134R) injected animals (Supplemental Figure 11). We secured a fiber-optic cannula guide to the surface of the thinned skull above M2 without puncturing the dura and therefore leaving the brain intact (Figure 4b), which we consider to be minimally invasive. Despite the presence of the highly optically scattering calavarial bone, stimulation with $20 \mathrm{~mW} 447 \mathrm{~nm}$ light induced left-turning behavior in animals expressing ChRger2 but not in animals expressing ChR2(H134R) (Figure 4b and Supplemental Video 1-2). Left-turning behavior terminated upon conclusion of optical stimulation (Supplemental Video 1). Behavioral effects were seen at powers as low as 10 $\mathrm{mW}$. To ensure that the turning behavior was not due to visual stimuli or heating caused by the stimulation laser, we repeated treadmill experiments using $671 \mathrm{~nm}$ light, which is outside the excitation spectrum of both opsins. $20 \mathrm{~mW} 671 \mathrm{~nm}$ light failed to induce turning in both ChRger2 and ChR2(H124R). Overall, these experiments demonstrate that ChRger2 is compatible with minimally-invasive systemic gene delivery and can enable minimallyinvasive optogenetic excitation. Coupling ChRgers with recently reported upconversion nanoparticles may allow for non-invasive optogenetics in deep brain areas with systemic transgene delivery and tissue-penetrating near-infrared (NIR) light for neuronal excitation ${ }^{29}$.

\section{Discussion}

We demonstrated a data-driven approach to engineering $\mathrm{ChR}$ properties that enables efficient discovery of highly functional $\mathrm{ChR}$ variants based on data from relatively few variants. In this approach we approximate the ChR fitness landscape for a set of $\sim 120,000$ chimeric ChRs and use it to efficiently search sequence space and select top-performing variants for a given property ${ }^{10,24,34}$. By first eliminating the vast majority of non-functional sequences, we can focus on local peaks scattered throughout the landscape. Then, using regression models, we predict which sequences lie on the fitness peaks.

Machine learning provides a platform for simultaneous optimization of multiple $\mathrm{ChR}$ properties that follow engineering specifications. We were able to generate variants with large variations in off-kinetics ( $10 \mathrm{~ms}$ to $>10 \mathrm{~s}$ ) and photocurrents that far exceed any of the parental or other commonly used ChRs. We also use the machine-learning models to identify the residues and contacts most important for $\mathrm{ChR}$ function. Application of this machinelearning pipeline (limited data collection from diverse sequences, model training and validation, and prediction and testing of new sequences) has great potential to optimize other 
neuroscience tools, e.g., anion-conducting $\mathrm{ChRs}^{12}$, calcium sensors, voltage sensors ${ }^{35}$, and $\mathrm{AAVs}^{30}$.

We designed high-performance ChRs (ChRger1-3) with unprecedented light sensitivity and validated ChRger2's application for in vivo optogenetics. The high-photocurrent properties of these ChRs overcome the limitation of low per-cell copy number after systemic delivery. ChRger2 enabled neuronal excitation with high temporal precision without invasive intracranial surgery for virus delivery or fiber optic implantation for superficial brain areas, extending what is currently possible for optogenetics experiments.

\section{Online methods}

\section{Construct design and cloning}

The design, construction, and characterization of the recombination library of chimeras is described in detail in Bedbrook et al. ${ }^{9}$. The 10-block contiguous and 10-block noncontiguous recombination libraries were designed and built using SCHEMA recombination ${ }^{9}$. Software packages for calculating SCHEMA energies are openly available at cheme.che.caltech.edu/groups/fha/Software.htm. Each chimeric ChR variant in these libraries is composed of blocks of sequence from the parental ChR $\left(\mathrm{CsChrimR}{ }^{11}, \mathrm{C} 1 \mathrm{C} 2^{17}\right.$, and CheRiff ${ }^{22}$ ), including chimeras with single-block swaps (chimeras consisting of 9 blocks of one parent and a single block from one of the other two parents) and multi-blockswap chimera sequences.

Selected ChR variant genes were inserted into a constant vector backbone [pFCK from Addgene plasmid \#51693 ${ }^{22}$ ] with a CMV promoter, Golgi export trafficking signal (TS) sequence (KSRITSEGEYIPLDQIDINV) ${ }^{5}$, and fluorescent protein (mKate). All ChR variants contain the SpyTag sequence following the N-terminal signal peptide for the SpyTag/SpyCatcher labeling assays used to characterize ChR membrane localization ${ }^{9,36}$. The $\mathrm{C} 1 \mathrm{C} 2$ parent for the recombination libraries is mammalian codon-optimized. ChR variant sequences used in this study are documented in Dataset 2. All selected ChR genes were synthesized and cloned in the $\mathrm{pFCK}$ mammalian expression vector by Twist Bioscience. For visualization, sequence alignment between $\mathrm{C} 1 \mathrm{C} 2$ and designer $\mathrm{ChRs}$ were created using Clustal $\Omega$ and visualized using ENDscript ${ }^{37}$ (Supplemental Figure 1c,d).

For characterization in neurons, selected ChR variants [ChRger1, ChRger2, ChRger3, CoChR ${ }^{11}$, and hChR2(H134R)] were inserted into a pAAV-hSyn vector backbone [Addgene plasmid \#26973], a pAAV-CamKIIa vector backbone [Addgene plasmid \#51087], and a pAAV-CAG-DIO vector backbone [Addgene plasmid \#104052]. In all backbones, each ChR was inserted with a TS sequence ${ }^{5}$ and fluorescent protein (eYFP).

\section{HEK293T cell and primary neuronal cultures}

The culturing and characterization ChRs in HEK cells is described in Bedbrook et al. ${ }^{9,36}$. Briefly, HEK cells were cultured at $37{ }^{\circ} \mathrm{C}$ and $5 \% \mathrm{CO}_{2}$ in D10 [DMEM supplemented with $10 \%$ (vol/vol) FBS, $1 \%$ sodium bicarbonate, and $1 \%$ sodium pyruvate]. HEK cells were transfected with purified ChR variant DNA using FuGENE®6 reagent according to the manufacturer's (Promega) recommendations. Cells were given 48 hours to express the ChRs 
before photocurrent measurements. Primary hippocampal neuronal cultures were prepped from C57BL/6N mouse embryos 16-18 days post-fertilization (E16-E18 Charles-River Labs) and cultured at $37{ }^{\circ} \mathrm{C}$ in the presence of $5 \% \mathrm{CO}_{2}$ in Neurobasal media supplemented with glutamine and B27. Cells were transduced 3-4 days after plating with rAAV-PHP.eB packaging ChR2(H134R), CoChR, ChRger1, ChRger2, or ChRger3. Whole-cell recordings were performed 5-10 days after transduction.

\section{Patch-clamp electrophysiology}

Whole-cell patch-clamp and cell-attached recordings were performed in transfected HEK cells, transduced cultured neurons, and acute brain slices to measure light-activated inward currents or neuronal firing. For electrophysiological recordings, cultured cells were continuously perfused with extracellular solution at room temperature (in mM: $140 \mathrm{NaCl}, 5$ $\mathrm{KCl}, 10$ HEPES, $2 \mathrm{MgCl}_{2}, 2 \mathrm{CaCl}_{2}, 10$ glucose; $\mathrm{pH}$ 7.35) while mounted on the microscope stage. For slice recordings, $32{ }^{\circ} \mathrm{C}$ artificial cerebrospinal fluid (ACSF) was continuously perfused over slices. ACSF contained $127 \mathrm{mM} \mathrm{NaCl}, 2.5 \mathrm{mM} \mathrm{KCl}, 25 \mathrm{mM} \mathrm{NaHCO}_{3}, 1.25$ $\mathrm{mM} \mathrm{NaH} \mathrm{PO}_{4}, 12 \mathrm{mM} d$-glucose, $0.4 \mathrm{mM}$ sodium ascorbate, $2 \mathrm{mM} \mathrm{CaCl}$, and $1 \mathrm{mM}$ $\mathrm{MgCl}_{2}$ and was bubbled continuously with $95 \%$ oxygen $/ 5 \% \mathrm{CO}_{2}$. Firing and photocurrent measurements were performed in the presence of $3 \mathrm{mM}$ kynurenic acid and $100 \mu \mathrm{M}$ picrotoxin to block optically evoked ionotropic glutamatergic and GABAergic currents, respectively.

Patch pipettes were fabricated from borosilicate capillary glass tubing (1B150-4; World Precision Instruments) using a model P-2000 laser puller (Sutter Instruments) to resistances of 3-6 M $\Omega$. Pipettes were filled with K-gluconate intracellular solution containing the following (in mM): $134 \mathrm{~K}$ gluconate, 5 EGTA, $10 \mathrm{HEPES}, 2 \mathrm{MgCl}_{2}, 0.5 \mathrm{CaCl}_{2}, 3 \mathrm{ATP}$, and 0.2 GTP. Whole-cell patch-clamp and cell-attached recordings were made using a Multiclamp 700B amplifier (Molecular Devices), a Digidata 1440 digitizer (Molecular Devices), and a PC running pClamp (version 10.4) software (Molecular Devices) to generate current injection waveforms and to record voltage and current traces.

Photocurrents were recorded from cells in voltage clamp held at $-60 \mathrm{mV}$. Neuronal firing was measured in current clamp mode with current injection for a $-60 \mathrm{mV}$ holding potential. Access resistance $\left(R_{\mathrm{a}}\right)$ and membrane resistance $\left(R_{\mathrm{m}}\right)$ were monitored throughout recording, and cells were discarded if $\mathrm{R}_{\mathrm{a}}$ or $\mathrm{R}_{\mathrm{m}}$ changed more than $15 \%$. During ChR variant functional screening in HEK cells, photocurrents were only recorded from cells that passed our recording criteria: $R_{m}>200 M \Omega$ and holding current $>-100$ pA. Our measured membrane properties of $\mathrm{ChR}$ expressing neurons were consistent with previous literature of opsin-expressing cells ${ }^{11}$ and are also consistent with previous reports of properties of cultured hippocampal neurons ${ }^{38,39}$ and PFC neurons in slice ${ }^{40,41}$ (Supplemental Figures 10). For cell culture experiments, the experimenter was blinded to the identity of the $\mathrm{ChR}$ being patched but not to the fluorescence level of the cells. For acute slice recordings, the experimenter was not blinded to the identity of the ChR. 


\section{Light delivery and imaging}

Patch-clamp recordings were done with short light pulses to measure photocurrents. Light pulse duration, wavelength, and power were varied depending on the experiment (as described in the text). Light pulses were generated using a Lumencor SPECTRAX light engine. The illumination/output spectra for each color were measured (Supplemental Figure 5). To evaluate normalized green photocurrent, we measured photocurrent strength at three wavelengths (peak \pm half width at half maximum): (red) $640 \pm 3 \mathrm{~nm}$, (green) $546 \pm 16 \mathrm{~nm}$, and (cyan) $481 \pm 3 \mathrm{~nm}$ with a $0.5 \mathrm{~s}$ light pulse. Light intensity was matched for these measurements, with $481 \mathrm{~nm}$ light at $2.3 \mathrm{~mW} \mathrm{~mm}^{-2}, 546 \mathrm{~nm}$ light at $2.8 \mathrm{~mW} \mathrm{~mm}^{-2}$, and 640 $\mathrm{nm}$ light at $2.2 \mathrm{~mW} \mathrm{~mm}^{-2}$. For full spectra measurements depicted in Figure 2e, we measured photocurrents at seven different wavelengths (peak \pm half width half maximum): (red) $640 \pm 3 \mathrm{~nm}$, (yellow) $567 \pm 13 \mathrm{~nm}$, (green) $546 \pm 16 \mathrm{~nm}$, (teal) $523 \pm 6 \mathrm{~nm}$, (cyan) 481 $\pm 3 \mathrm{~nm}$ LED, (blue) $439 \pm 8 \mathrm{~nm} \mathrm{LED}$, and (violet) $397 \pm 3 \mathrm{~nm}$ with a $0.5 \mathrm{~s} \mathrm{light}$ pulse for each color. Light intensity is matched across wavelengths at $1.3 \mathrm{~mW} \mathrm{~mm}^{-2}$.

Imaging of ChR variants expression in HEK cells was performed using an Andor Neo 5.5 sCMOS camera and Micro-Manager Open Source Microscopy Software. Imaging of ChR expression in neuronal cultures and in brain slices was performed using a Zeiss LSM 880 confocal microscope and Zen software.

\section{Electrophysiology data analysis}

Electrophysiology data were analyzed using Clampfit 10.7 (Molecular Devices, LLC) and custom data-processing scripts written using open-source packages in the Python programming language to perform baseline adjustments, find the peak and steady state inward currents, perform monoexponential fits of photocurrent decay for off-kinetic properties, and quantify spike fidelity. Only neurons with an uncompensated series resistance between 5 and $25 \mathrm{M} \Omega, R_{\mathrm{m}}>90 \mathrm{M} \Omega$, and holding current $>-150 \mathrm{pA}$ (holding at $-60 \mathrm{mV}$ ) were included in data analysis (Supplemental Figures 10). The photocurrent amplitude was not adjusted for expression level since both expression and conductance contribute to the in vivo utility of the tool. However, comparisons of expression with photocurrent strength for all ChR variants tested are included in Supplemental Figures 6-7. As metrics of photocurrent strength, peak and steady-state photocurrent were used (Figure 1a). As a metric for the ChR activation spectrum, the normalized current strength induced by exposure to green light (546 nm) was used (Figure 1a). Two parameters were used to characterize ChR off-kinetics: the time to reach $50 \%$ of the light-activated current and the photocurrent decay rate, $\tau_{\text {off }}$ (Figure 1a).

\section{AAV production and purification}

Production of recombinant AAV-PHP.eB packaging pAAV-hSyn-X-TS-eYFP-WPRE, pAAV-CAG-DIO[X-TS-eYFP]-WPRE, and pAAV-CaMKIIa-X-TS-eYFP-WPRE (X = ChR2(H134R), CoChR, ChRger1, ChRger2, and ChRger3) was done following the methods described in Deverman et al. ${ }^{42}$ and Challis et al. ${ }^{31}$. Briefly, triple transfection of HEK293T cells (ATCC) was performed using polyethylenimine (PEI). Viral particles were harvested from the media and cells. Virus was then purified over iodixanol (Optiprep, Sigma; D1556) step gradients $(15 \%, 25 \%, 40 \%$ and $60 \%)$. Viruses were concentrated and formulated in 
phosphate buffered saline (PBS). Virus titers were determined by measuring the number of DNase I-resistant viral genomes using qPCR with linearized genome plasmid as a standard.

\section{Animals}

All procedures were approved by the California Institute of Technology Institutional Animal Care and Use Committee (IACUC). Dat-Cre mice (006660) and C57B1/6J mice (000664) were purchased from Jackson Laboratory.

\section{Intravenous injections, stereotactic injections, and cannula implantation}

Intravenous administration of rAAV vectors was performed by injecting the virus into the retro-orbital sinus at viral titers indicated in the text. There were no observed health issues with animals after systemic injection of virus at the titers presented in the paper. Mice remain healthy $>6$ months after systemic delivery of ChR2 and ChRgers. With slice electrophysiology, there was no observed indication of poor cell health due to viral-mediated expression, which was quantified by measuring the membrane resistance $\left[R_{\mathrm{m}}\right]$, leak current [holding at $-60 \mathrm{mV}$ ], and resting membrane potential (Supplemental Figures 10). Local expression in the prefrontal cortex (PFC) was achieved by direct stereotactic injection of $1 \mu \mathrm{l}$ of purified AAV vectors at $5 \times 10^{12} \mathrm{vg} \mathrm{ml}^{-1}$ targeting the following coordinates: anteriorposterior (AP), -1.7 ; media-lateral (ML), $+/-0.5$; and dorsal-ventral (DV), -2.2. For stimulation of the VTA, $300 \mu \mathrm{m}$ outer diameter mono fiber-optic cannulae (Doric Lenses, MFC_300/330-0.37_6mm_ZF1.25_FLT) were stereotaxically implanted $200 \mu \mathrm{m}$ above the VTA bilaterally targeted to the following coordinates: AP, $-3.44 \mathrm{~mm}$; ML, $+/-0.48 \mathrm{~mm}$; DV, $4.4 \mathrm{~mm}$. For stimulation of the right secondary motor cortex (M2), $3 \mathrm{~mm}$ long, $400 \mu \mathrm{m}$ mono fiber-optic cannulae (Doric Lenses, MFC_400/430-0.48_3mm_ZF1.25_FLT) were surgically secured to the surface of the skull above M2 (unilaterally) targeted to the following coordinates: AP, $1 \mathrm{~mm}$; ML, $0.5 \mathrm{~mm}$. The skull was thinned $~ 40-50 \%$ with a standard drill to create a level surface for the fiber-skull interface. Light was delivered from either a $447 \mathrm{~nm}$ or $671 \mathrm{~nm}$ laser (Changchun New Industries [CNI] Model with PSU-HLED) via mono fiber-optic patch cable(s) (Doric Lenses, MFP_400/430/1100-0.48_2m_FCZF1.25) coupled to the fiber-optic cannula(e). Fiber-optic cannulae were secured to the skull with Metabond (Parkel, SKU S396) and dental cement.

Analysis of behavioral experiments was performed using the open-source MATLAB program OptiMouse ${ }^{43}$ to track mouse nose, body, and tail position while the mouse was running on the treadmill. Optogenetic intracranial self-stimulation was performed using a mouse modular test chamber (Lafayette Instruments, Model 80015NS) outfitted with an IR nose port (Model 80116TM).

\section{Gaussian process modeling}

Both the GP regression and classification modeling methods applied in this paper are based on work detailed in ref 8 and 23. For modeling, all sequences were aligned using MUltiple Sequence Comparison by Log-Expectation (MUSCLE) (https://www.ebi.ac.uk/Tools/msa/ muscle/). For modeling, aligned sequences were truncated to match the length of the $\mathrm{C} 1 \mathrm{C} 2$ sequence, eliminating $\mathrm{N}$ - and $\mathrm{C}$-terminal fragments with poor alignment quality due to high sequence diversity (Dataset 1 and Dataset 2). Structural encodings (i.e., the contact map) use 
the $\mathrm{C} 1 \mathrm{C} 2$ crystal structure (3UG9.pdb) and assume that $\mathrm{ChR}$ chimeras share the contact architecture observed in the $\mathrm{C} 1 \mathrm{C} 2$ crystal structure. Models built using structural encodings built from the ChR2 structure (6EID.pdb) and the C1Chrimson structure (5ZIH.pdb) performed as well as models using the $\mathrm{C} 1 \mathrm{C} 2$ structure (Supplemental Figure 1c,d). The models are robust to differences in contact maps because they use both sequence and structural information, which is somewhat redundant.

For a given ChR, the contact map is simply a list of contacting amino acids with their positions. For example, a contact between alanine at position 134 and methionine at position 1 of the amino acid sequence would be encoded by [('A134'), ('M1')]. Both sequence and structural information were one-hot encoded. Regression models for ChR properties were trained to predict the logarithm of the measured properties. All training data was normalized to have mean zero and standard deviation one.

Gaussian process regression and classification models require kernel functions that measure the similarity between protein sequences. Learning involves optimizing the form of the kernel and its hyperparameters (Supplemental Table 2). The Matérn kernel was found to be optimal for all ChR properties (Supplemental Table 1).

For classification model training, all 102 functionally characterized $\mathrm{ChR}$ variants from our recombination libraries (Dataset 2) were used as well as data from 61 sequence variants published by others (Dataset 1 ). The model was then updated with data collected from the 22 additional $\mathrm{ChR}$ recombination variants with high sequence diversity ( 70 mutations from the closest parent) and predicted to be functional (Figure 1d). For training the regression models, all 102 functionally characterized training sequences (Dataset 2) were initially used and then the models were updated with data collected from the 22 additional ChR variants (Figure 1d).

GP regression-In regression, the goal is to infer the value of an unknown function $f(x)$ at a novel point $x_{*}$ given observations $y$ at inputs $X$. Assuming that the observations are subject to independent and identically distributed Gaussian noise with variance $\sigma_{n}^{2}$, the posterior distribution of $f_{*}=f\left(x_{*}\right)$ for Gaussian process regression is Gaussian with mean

$$
\bar{f}_{*}=k_{*}^{T}\left(K+\sigma_{n}^{2} I\right)^{-1} y
$$

and variance

$$
\nu_{*}=k\left(x_{*}, x_{*}\right)-k_{*}^{T}\left(K+\sigma_{n}^{2} I\right)^{-1} k_{*}
$$

Where $K$ is the symmetric, square covariance matrix for the training set: $K_{i j}=k\left(x_{i}, x_{j}\right)$ for $x_{i}$ and $x_{j}$ in the training set. $k_{*}$ is the vector of covariances between the novel input and each input in the training set, and $k_{*_{i}}=k\left(x_{*}, x_{i}\right)$. The hyperparameters in the kernel functions and the noise hyperparameter $\sigma_{n}$ were determined by maximizing the log marginal likelihood: 


$$
\log p(y \mid X)=-\frac{1}{2} y^{T}\left(K+\sigma_{n}^{2} I\right)^{-1} y-\frac{1}{2} \log \left|K+\sigma_{n}^{2} I\right|-\frac{n}{2} \log 2 \pi
$$

where $n$ is the dimensionality of the inputs. Regression was implemented using open-source packages in the SciPy ecosystem ${ }^{44-46}$.

GP classification-In binary classification, instead of continuous outputs $y$, the outputs are class labels $y_{i} \in\{+1,-1\}$, and the goal is to use the training data to make probabilistic predictions $\pi\left(x_{*}\right)=p\left(y_{*}=+1 \mid x_{*}\right)$. We use Laplace's method to approximate the posterior distribution. Hyperparameters in the kernels are found by maximizing the marginal likelihood. Classification was implemented using open-source packages in the SciPy ecosystem ${ }^{44-46}$. The binary classification model was trained to predict if a ChR sequence is or is not functional. A ChR sequence was considered to be functional if its photocurrents were $>100$ pA upon light exposure, a threshold set as an approximate lower bound for current necessary for neuronal activation.

GP kernels for modeling proteins-Gaussian process regression and classification models require kernel functions that measure the similarity between protein sequences. A protein sequence $s$ of length $L$ is defined by the amino acid present at each location. This can be encoded as a binary feature vector $x_{S e}$ that indicates the presence or absence of each amino acid at each position resulting in a vector of length $20 L$ (for 20 possible amino acids). Likewise, the protein's structure can be represented as a residue-residue contact map. The contact map can be encoded as a binary feature vector $x_{s t}$ that indicates the presence or absence of each possible contacting pair. Both the sequence and structure feature vectors were used by concatenating them to form a sequence-structure feature vector.

Three types of kernel functions $k\left(s_{i}, s_{j}\right)$ were considered: polynomial kernels, squared exponential kernels, and Matérn kernels. These different forms represent possible functions for the protein's fitness landscape. The polynomial kernel is defined as:

$$
k\left(s, s^{\prime}\right)=\left(\sigma_{0}^{2}+\sigma_{p}^{2} x^{T} x^{\prime}\right)^{d}
$$

where $\sigma_{0}$ and $\sigma_{p}$ are hyperparameters. We considered polynomial kernels with $d=3$. The squared exponential kernel is defined as:

$$
k\left(s, s^{\prime}\right)=\sigma_{p}^{2} \exp \left(-\frac{\left\|x-x^{\prime}\right\|_{2}^{2}}{l}\right)
$$

where 1 and $\sigma_{p}$ are also hyperparameters and $|\cdot|_{2}$ is the L2 norm. Finally, the Matérn kernel with $v=\frac{5}{2}$ is defined as:

$$
k\left(s, s^{\prime}\right)=\left(1+\frac{\sqrt{5\left\|x-x^{\prime}\right\|_{2}^{2}}}{l}+\frac{5\left\|x-x^{\prime}\right\|_{2}^{2}}{3 l^{2}}\right) \exp \left(-\frac{\sqrt{5\left\|x-x^{\prime}\right\|_{2}^{2}}}{l}\right)
$$


Where $I$ is once again a hyperparameter.

L1 regression feature identification and weighting - L1 regression was used to identify residues and contacts in the ChR structure most important for each ChR functional property of interest. First, residues and contacts that covary were identified using the concatenated sequence and structure binary feature vector for each of the training set $\mathrm{ChR}$ variants. Each set of covarying residues and contacts was combined into a single feature. L1 linear regression was used to select the features that contribute most to each $\mathrm{ChR}$ functional property of interest. The level of regularization was chosen by maximizing the log marginal likelihood of the Gaussian process regression model trained on the features selected at that level of regularization. We then performed Bayesian ridge regression on the selected features using the default settings in scikit-learn ${ }^{47}$. Residues and contacts with the largest absolute Bayesian ridge linear regression weights were plotted onto the $\mathrm{C} 1 \mathrm{C} 2$ structure (Supplemental Figure 4). For feature identification and weighting, models were trained on both the training set and also the test set of $28 \mathrm{ChR}$ sequences predicted to have useful combinations of diverse properties.

\section{Statistical analysis}

Plotting and statistical analysis were done in Python 2.7 and 3.6 and GraphPad Prism 7.01. For statistical comparisons, we first performed a D'Agostino \& Pearson normality test. If the $p$-value of a D'Agostino \& Pearson normality test was < 0.05 , the non-parametric KruskalWallis test with Dunn's multiple comparisons post hoc test was used. If the data passed the normality test, a one-way ANOVA was used.

\section{Data availability}

The authors declare that data supporting the findings of this study are available within the paper and its supplementary information files. Source data for classification model training are provided in Dataset 1 and Dataset 2. Source data for regression model training are provided in Dataset 2. DNA constructs for the ChRger variants are deposited for distribution at Addgene (http://www.addgene.org, plasmid numbers 127237-44).

\section{Code availability}

Code used to train classification and regression models can be found at: https://github.com/ fhalab/channels.

\section{Supplementary Material}

Refer to Web version on PubMed Central for supplementary material.

\section{Acknowledgements}

We thank Twist Bioscience for synthesizing and cloning ChR sequences, Dr. Daniel Wagenaar and the Caltech Neurotechnology Center for building the mouse treadmill, Joshua Brake for performing spectrometer measurements, Dr. John Bedbrook for critical reading of the manuscript, and the Gradinaru and Arnold labs for helpful discussions. This work was funded by the National Institute of Health (V.G.), the Institute for Collaborative Biotechnologies grant W911NF-09-0001 from the U.S. Army Research Office (F.H.A), NIH BRAIN R01MH117069, NIH Director's Pioneer Award DP1OD025535, NIH Director's New Innovator Award DP2NS087949, and SPARC OT2OD023848. Additional funding includes the NSF NeuroNex Technology Hub 
grant 1707316 (V.G.), the CZI Neurodegeneration Challenge Network (V.G.), the Vallee Foundation (V.G.), the Heritage Medical Research Institute (V.G.), and the Beckman Institute for CLARITY, Optogenetics and Vector Engineering Research for technology development and broad dissemination: clover.caltech.edu (V.G.). The content of the information does not necessarily reflect the position or the policy of the Government, and no official endorsement should be inferred. C.N.B. is funded by Ruth L. Kirschstein National Research Service Awards F31MH102913. J.E.R. is supported by the Children's Tumor Foundation (Young Investigator Award 2016-01-006).

\section{References}

1. Deisseroth K \& Hegemann P The form and function of channelrhodopsin. Science 357, doi:10.1126/ science.aan5544 (2017).

2. Yizhar O, Fenno LE, Davidson TJ, Mogri M \& Deisseroth K Optogenetics in neural systems. Neuron 71, 9-34, doi:10.1016/j.neuron.2011.06.004 (2011). [PubMed: 21745635]

3. Lin JY A user's guide to channelrhodopsin variants: features, limitations and future developments. Experimental physiology 96, 19-25, doi:10.1113/expphysiol.2009.051961 (2011). [PubMed: 20621963]

4. Zhang F et al. Optogenetic interrogation of neural circuits: technology for probing mammalian brain structures. Nature protocols 5, 439-456, doi:10.1038/nprot.2009.226 (2010). [PubMed: 20203662]

5. Gradinaru V et al. Molecular and cellular approaches for diversifying and extending optogenetics. Cell 141, 154-165, doi:10.1016/j.cell.2010.02.037 (2010). [PubMed: 20303157]

6. Mattis $\mathrm{J}$ et al. Principles for applying optogenetic tools derived from direct comparative analysis of microbial opsins. Nature methods 9, 159-172, doi:10.1038/nmeth.1808 (2011). [PubMed: 22179551]

7. Chuong AS et al. Noninvasive optical inhibition with a red-shifted microbial rhodopsin. Nature neuroscience 17, 1123-1129, doi:10.1038/nn.3752 (2014). [PubMed: 24997763]

8. Bedbrook CN, Yang KK, Rice AJ, Gradinaru V \& Arnold FH Machine learning to design integral membrane channelrhodopsins for efficient eukaryotic expression and plasma membrane localization. PLoS computational biology 13, e1005786, doi:10.1371/journal.pcbi.1005786 (2017). [PubMed: 29059183]

9. Bedbrook CN et al. Structure-guided SCHEMA recombination generates diverse chimeric channelrhodopsins. Proceedings of the National Academy of Sciences of the United States of America (2017).

10. Romero PA \& Arnold FH Exploring protein fitness landscapes by directed evolution. Nature reviews. Molecular cell biology 10, 866-876, doi:10.1038/nrm2805 (2009). [PubMed: 19935669]

11. Klapoetke NC et al. Independent optical excitation of distinct neural populations. Nature methods 11, 338-346, doi:10.1038/nmeth.2836 (2014). [PubMed: 24509633]

12. Govorunova EG, Sineshchekov OA, Janz R, Liu X \& Spudich JL Natural light-gated anion channels: A family of microbial rhodopsins for advanced optogenetics. Science 349, 647-650, doi: 10.1126/science.aaa7484 (2015). [PubMed: 26113638]

13. Lin JY, Knutsen PM, Muller A, Kleinfeld D \& Tsien RY ReaChR: a red-shifted variant of channelrhodopsin enables deep transcranial optogenetic excitation. Nature neuroscience 16, 14991508, doi:10.1038/nn.3502 (2013). [PubMed: 23995068]

14. Berndt A, Yizhar O, Gunaydin LA, Hegemann P \& Deisseroth K Bi-stable neural state switches. Nature neuroscience 12, 229-234, doi:10.1038/nn.2247 (2009). [PubMed: 19079251]

15. Lin JY, Lin MZ, Steinbach P \& Tsien RY Characterization of engineered channelrhodopsin variants with improved properties and kinetics. Biophysical journal 96, 1803-1814, doi:10.1016/ j.bpj.2008.11.034 (2009). [PubMed: 19254539]

16. Berndt A et al. Structural foundations of optogenetics: Determinants of channelrhodopsin ion selectivity. Proc Natl Acad Sci U S A 113, 822-829, doi:10.1073/pnas.1523341113 (2016). [PubMed: 26699459]

17. Kato HE et al. Crystal structure of the channelrhodopsin light-gated cation channel. Nature 482, 369-374, doi:10.1038/nature10870 (2012). [PubMed: 22266941]

18. Wietek $\mathrm{J}$ et al. Conversion of channelrhodopsin into a light-gated chloride channel. Science 344 , 409-412, doi:10.1126/science.1249375 (2014). [PubMed: 24674867] 
19. Chan KY et al. Engineered AAVs for efficient noninvasive gene delivery to the central and peripheral nervous systems. Nature neuroscience 20, 1172-1179, doi:10.1038/nn.4593 (2017). [PubMed: 28671695]

20. Smith MA, Romero PA, Wu T, Brustad EM \& Arnold FH Chimeragenesis of distantly-related proteins by noncontiguous recombination. Protein science 22, 231-238, doi:10.1002/pro.2202 (2013). [PubMed: 23225662]

21. Voigt CA, Martinez C, Wang ZG, Mayo SL \& Arnold FH Protein building blocks preserved by recombination. Nature structural biology 9, 553-558, doi:10.1038/nsb805 (2002). [PubMed: 12042875]

22. Hochbaum DR et al. All-optical electrophysiology in mammalian neurons using engineered microbial rhodopsins. Nature methods 11, 825-833, doi:10.1038/nmeth.3000 (2014). [PubMed: 24952910]

23. Gunaydin LA et al. Ultrafast optogenetic control. Nature neuroscience 13, 387-392, doi: 10.1038/nn.2495 (2010). [PubMed: 20081849]

24. Romero PA, Krause A \& Arnold FH Navigating the protein fitness landscape with Gaussian processes. Proc Natl Acad Sci U S A 110, E193-201, doi:10.1073/pnas.1215251110 (2013). [PubMed: 23277561]

25. Volkov O et al. Structural insights into ion conduction by channelrhodopsin 2 . Science 358 , doi: 10.1126/science.aan8862 (2017).

26. Oda K et al. Crystal structure of the red light-activated channelrhodopsin Chrimson. Nature communications 9, 3949, doi:10.1038/s41467-018-06421-9 (2018).

27. Bamann C, Gueta R, Kleinlogel S, Nagel G \& Bamberg E Structural guidance of the photocycle of channelrhodopsin-2 by an interhelical hydrogen bond. Biochemistry 49, 267-278, doi:10.1021/ bi901634p (2010). [PubMed: 20000562]

28. Nagel G et al. Light activation of channelrhodopsin-2 in excitable cells of Caenorhabditis elegans triggers rapid behavioral responses. Current biology : CB 15, 2279-2284, doi:10.1016/j.cub. 2005.11.032 (2005). [PubMed: 16360690]

29. Chen $\mathrm{S}$ et al. Near-infrared deep brain stimulation via upconversion nanoparticle-mediated optogenetics. Science 359, 679-684, doi:10.1126/science.aaq1144 (2018). [PubMed: 29439241]

30. Bedbrook CN, Deverman BE \& Gradinaru V Viral Strategies for Targeting the Central and Peripheral Nervous Systems. Annual review of neuroscience 41, 323-348, doi:10.1146/annurevneuro-080317-062048 (2018).

31. Challis RC et al. Systemic AAV vectors for widespread and targeted gene delivery in rodents. Nature protocols, doi:10.1038/s41596-018-0097-3 (2019).

32. Pascoli V, Terrier J, Hiver A \& Luscher C Sufficiency of Mesolimbic Dopamine Neuron Stimulation for the Progression to Addiction. Neuron 88, 1054-1066, doi:10.1016/j.neuron. 2015.10.017 (2015). [PubMed: 26586182]

33. Gradinaru V et al. Targeting and readout strategies for fast optical neural control in vitro and in vivo. The Journal of neuroscience : the official journal of the Society for Neuroscience 27, 1423114238, doi:10.1523/JNEUROSCI.3578-07.2007 (2007). [PubMed: 18160630]

34. Yang KK, Wu Z \& Arnold FH Machine-learning-guided directed evolution for protein engineering. Nature methods, doi:10.1038/s41592-019-0496-6 (2019).

35. Flytzanis NC et al. Archaerhodopsin variants with enhanced voltage-sensitive fluorescence in mammalian and Caenorhabditis elegans neurons. Nature communications 5, 4894, doi:10.1038/ ncomms5894 (2014).

36. Bedbrook CN et al. Genetically Encoded Spy Peptide Fusion System to Detect Plasma MembraneLocalized Proteins In Vivo. Chemistry \& biology 22, 1108-1121, doi:10.1016/j.chembiol. 2015.06.020 (2015). [PubMed: 26211362]

37. Robert X \& Gouet P Deciphering key features in protein structures with the new ENDscript server. Nucleic acids research 42, W320-324, doi:10.1093/nar/gku316 (2014). [PubMed: 24753421]

38. Fan J et al. Reduced Hyperpolarization-Activated Current Contributes to Enhanced Intrinsic Excitability in Cultured Hippocampal Neurons from $\operatorname{PrP}(-/-)$ Mice. Frontiers in cellular neuroscience 10, 74, doi:10.3389/fncel.2016.00074 (2016). [PubMed: 27047338] 
39. Slomowitz E et al. Interplay between population firing stability and single neuron dynamics in hippocampal networks. eLife 4, doi:10.7554/eLife.04378 (2015).

40. Kroon T, van Hugte E, van Linge L, Mansvelder HD \& Meredith RM Early postnatal development of pyramidal neurons across layers of the mouse medial prefrontal cortex. Scientific reports 9, 5037, doi:10.1038/s41598-019-41661-9 (2019). [PubMed: 30911152]

41. van Aerde KI \& Feldmeyer D Morphological and physiological characterization of pyramidal neuron subtypes in rat medial prefrontal cortex. Cerebral cortex 25, 788-805, doi:10.1093/cercor/ bht278 (2015). [PubMed: 24108807]

42. Deverman BE et al. Cre-dependent selection yields AAV variants for widespread gene transfer to the adult brain. Nature biotechnology 34, 204-209, doi:10.1038/nbt.3440 (2016).

43. Ben-Shaul Y OptiMouse: a comprehensive open source program for reliable detection and analysis of mouse body and nose positions. BMC biology 15, 41, doi:10.1186/s12915-017-0377-3 (2017). [PubMed: 28506280]

44. Walt S, Colbert SC \& Varoquaux G The NumPy array: a structure for efficient numerical computation. Computing in Science and Engineering 13, 22-30 (2011).

45. Hunter JD Matplotlib: A 2D Graphics Environment. Computing in Science and Engineering 9, 9095 (2007).

46. Oliphant TE Python for Scientific Computing. Computing in Science and Engineering 9, 10-20 (2007).

47. Pedregosa F et al. Scikit-learn: Machine Learning in Python. Journal of Machine Learning Research 12, 2825-2830 (2011). 
a Photocurrent properties Current strength<smiles>C[C+]1CCC1</smiles>
Oft-kinetics

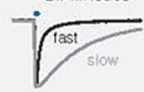

Wavelength sensitivity of activation

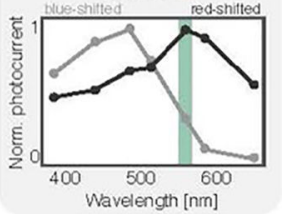

e
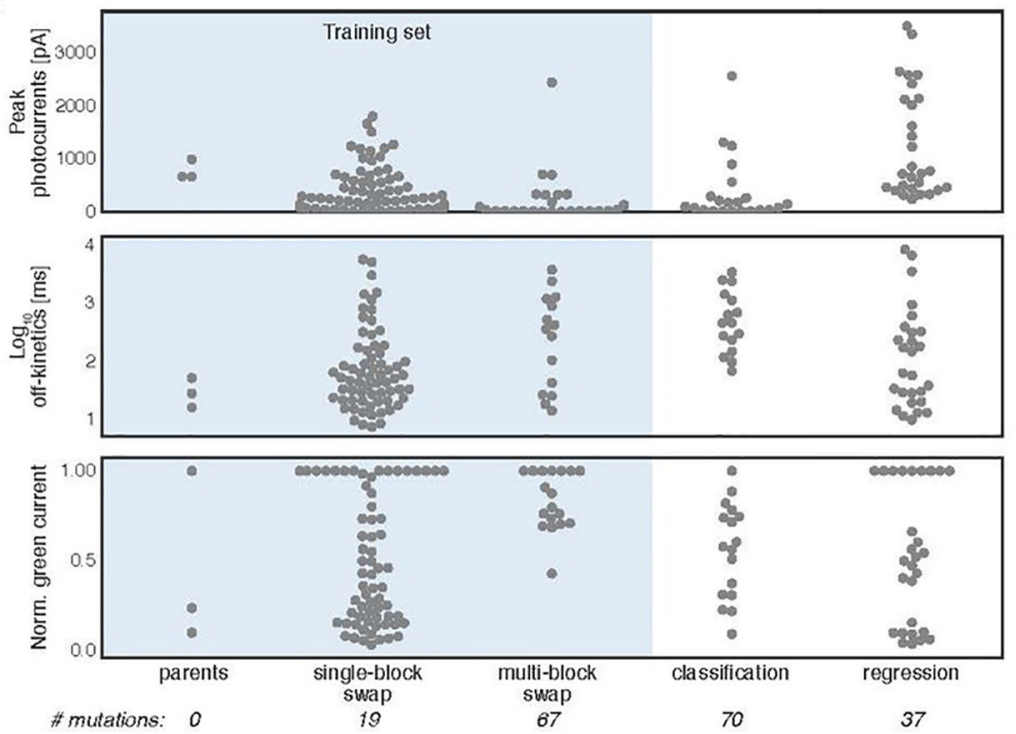
threshold out non-functional ChRs from the library

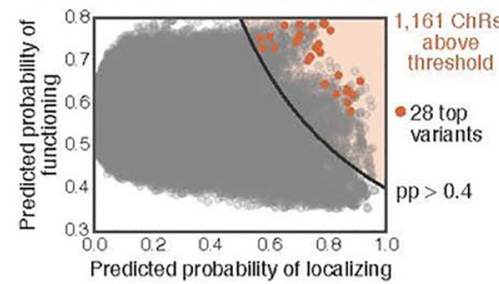

d Traning data

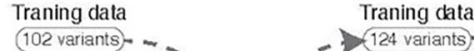
$(102$ variants) - -1 b

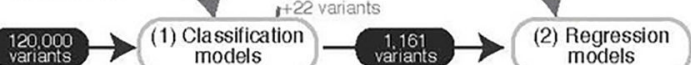

Figure 1. time to reach $50 \%$ of the light-exposed current after light removal. As a metric for ChRs above the 0.4 threshold for the product of the predicted probabilities (pp) of c approximate the fitness landscape for different properties
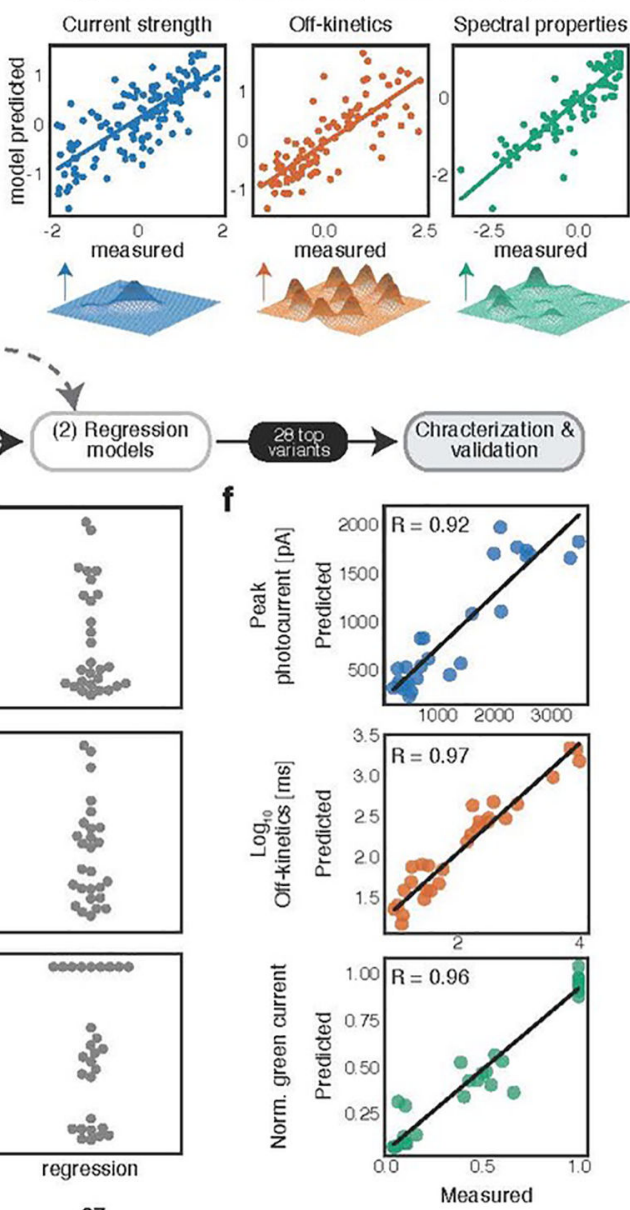

Machine learning-guided optimization of ChRs. (a) Upon light exposure, ChRs open and reach a peak inward current and then desensitize reaching a lower steady-state current. We use both peak and steady-state current as metrics for photocurrent strength. To evaluate ChR off-kinetics we used the current decay rate $\left(\tau_{\text {off }}\right)$ after a $1 \mathrm{~ms}$ light exposure and also the wavelength sensitivity of activation, we used the normalized photocurrent with green (546 $\mathrm{nm}$ ) light, which easily differentiates blue-shifted ChRs (peak activation: $450-480 \mathrm{~nm}$ ) and red-shifted ChRs (peak activation: $\sim 520-650 \mathrm{~nm}$ ). (b) We trained classification models to predict whether ChRs would localize correctly to the plasma membrane and function (i.e., localization and function). (c) We then trained regression models to approximate the fitness landscape for each property of interest for the recombination library (inset show hypothetical fitness landscapes). (b-c) Models were trained with photocurrent properties for each ChR in the training set (plots show 20-fold cross validation on the training set). Sequences predicted to localize and function by the classification models and predicted to have an optimized set of functional properties by the regression models were selected for further characterization, 
e.g., the 28 top variants. (d) The classification model was trained with 102 recombination variants (Dataset 2) and 61 previously-published ChRs (Dataset 1) and the regression models were trained with 124 recombination variants. (e) Measurements of training set ChR and model-predicted $\mathrm{ChR}$, peak photocurrent, off-kinetics, and normalized green current ( $n$ = 3-8 cells per variant; Dataset 2). Each gray-colored point is a ChR variant. Training set data are shaded in blue. Mean number of mutations for each set is below the plots. (f) Model predictions vs measured photocurrent property for each of the 28 designer ChRs. R represents the Pearson correlation coefficient. 
a

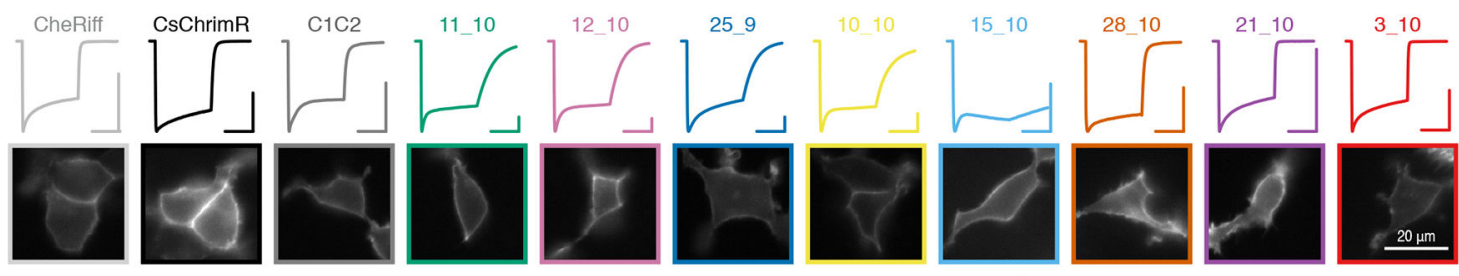

b
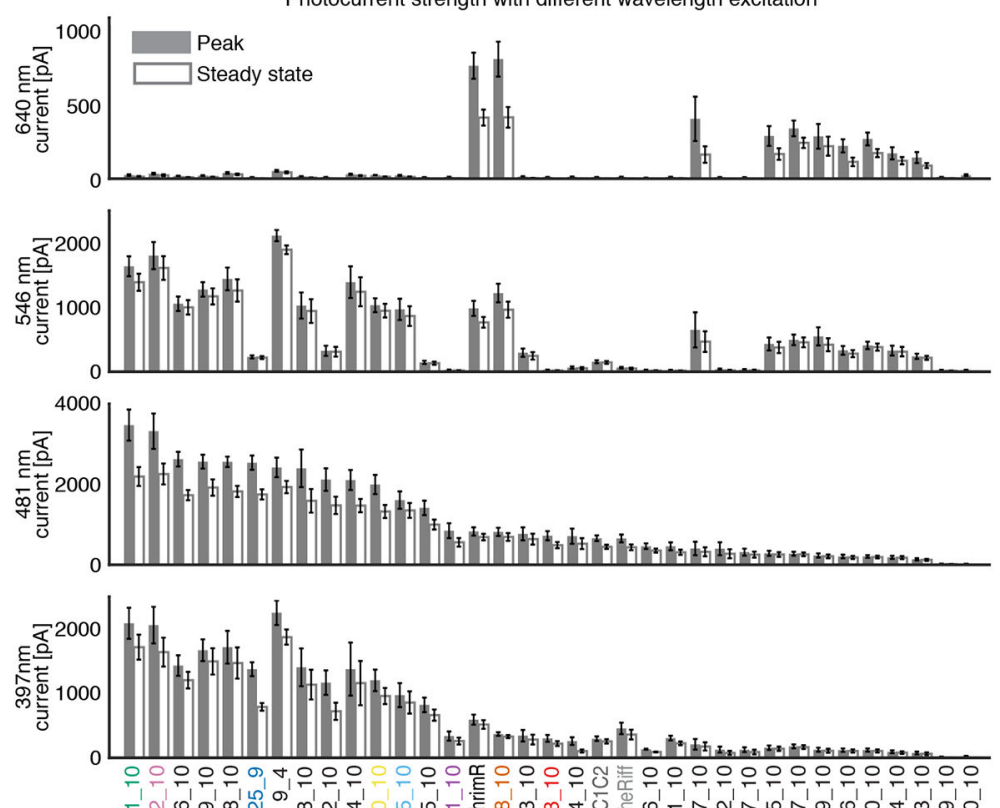

F⿻上,

d

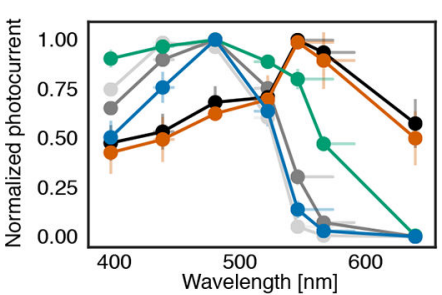

e

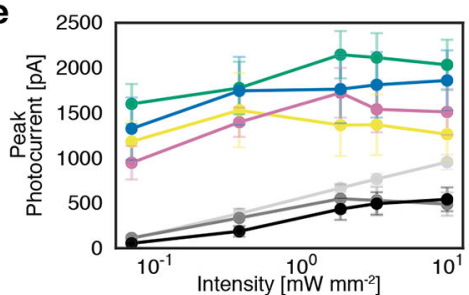

C

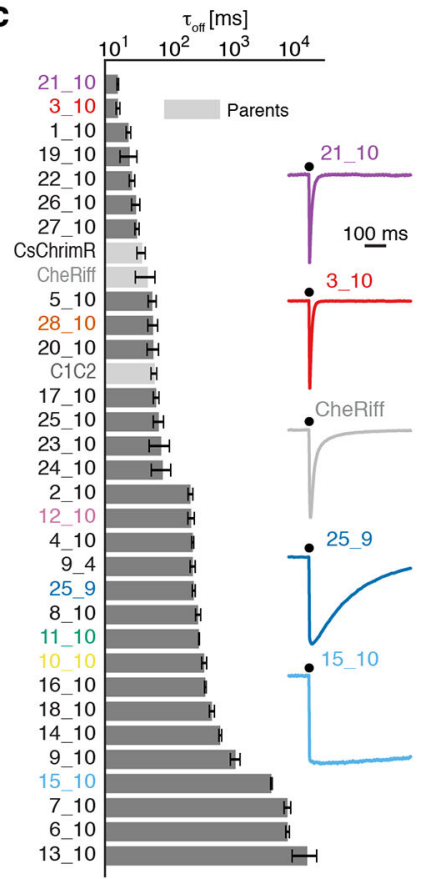

Figure 2.

The model-predicted ChRs exhibit a large range of functional properties often far exceeding the parents. (a) Representative current traces after $0.5 \mathrm{~s}$ light exposure for select designer ChR variants with corresponding expression and localization in HEK cells. Vertical colored scale bar for each $\mathrm{ChR}$ current trace represents $500 \mathrm{pA}$, and horizontal scale bar represents $250 \mathrm{~ms}$. The variant color presented in (a) is constant throughout panels. (b) Measured peak and steady-state photocurrent with different wavelengths of light in HEK cells $(n=4-8$ cells, see Dataset 2). $397 \mathrm{~nm}$ light at $1.5 \mathrm{~mW} \mathrm{~mm}^{-2}, 481 \mathrm{~nm}$ light at $2.3 \mathrm{~mW} \mathrm{~mm}^{-2}, 546 \mathrm{~nm}$ light at $2.8 \mathrm{~mW} \mathrm{~mm}^{-2}$, and $640 \mathrm{~nm}$ light at $2.2 \mathrm{~mW} \mathrm{~mm}^{-2}$. (c) Off-kinetics decay rate $\left(\tau_{\mathrm{off}}\right)$ following a $1 \mathrm{~ms}$ exposure to $481 \mathrm{~nm}$ light at $2.3 \mathrm{~mW} \mathrm{~mm}^{-2}(n=4-8$ cells, see Dataset 2). Parent ChRs are highlighted in light gray. Inset shows representative current traces with 1 ms light exposure for select ChRs revealing distinct profiles: ChR_21_10 turns off rapidly, ChR_25_9 and ChR_11_10 turn off more slowly, and ChR_15_10 exhibits little decrease in photocurrent $0.5 \mathrm{~s}$ after the light exposure. (d) Peak and steady-state photocurrent strength 
with varying light irradiances compared with parental ChRs (CheRiff, $n=5$ cells; CsChrimR, $n=5$ cells; C1C2, $n=4$ cells; $28 \_10, n=5$ cells; $11 \_10, n=5$ cells; 25_9, $n=5$ cells). (e) Wavelength sensitivity of activation for select ChRs compared with parental ChRs (CheRiff, $n=6$ cells; CsChrimR, $n=5$ cells; C1C2, $n=4$ cells; 11_10, $n=6$ cells; 12_10, $n$ $=7$ cells; 25_9, $n=5$ cells; $10 \_10, n=4$ cells). Top variants, ChR_9_4, ChR_25_9, and ChR_11_10 are named ChRger1, ChRger2, and ChRger3 in subsequent figures. Plotted data are mean \pm SEM. 
a

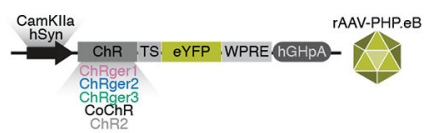

b

g

h

Figure 3.
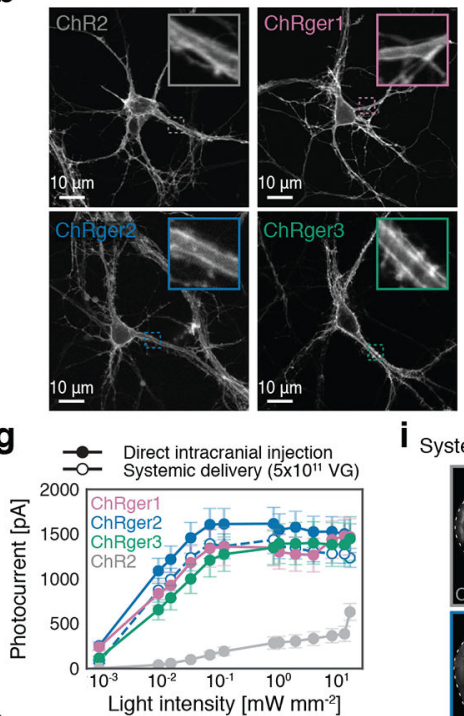

I Systemic delivery (5x10" vG)

C
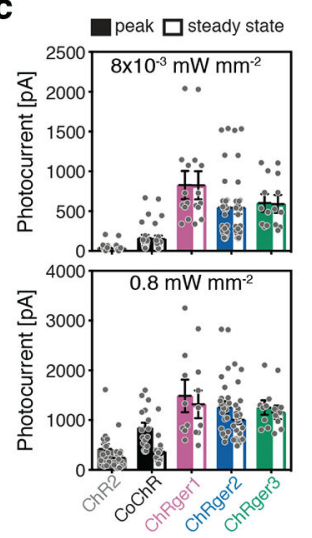
Light intensity

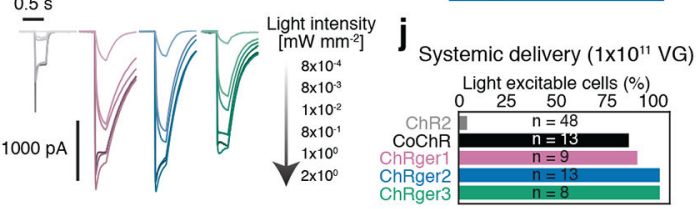

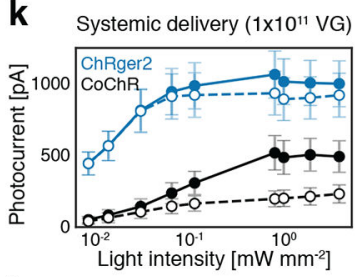

d

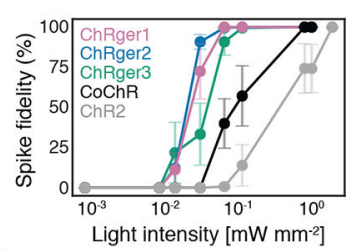

e

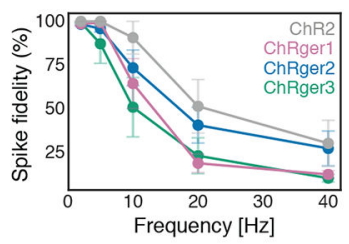

Light intensity:
$3 \times 10^{-2}\left[\mathrm{~mW} \mathrm{~mm}^{-2}\right]$

$50 \mathrm{mv}$ ChR2

CoChR

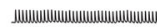

ChRger1

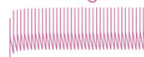

ChRger2

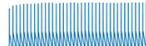

ChRger3

2

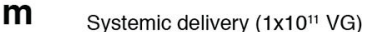

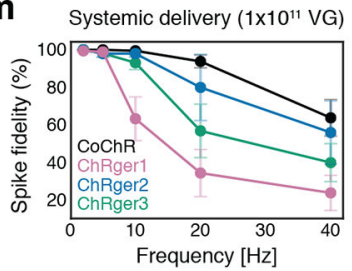

n ChRger1 ChRger2 ChRger3

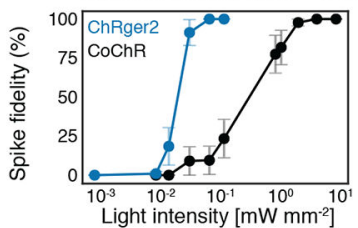

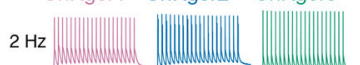

$5 \mathrm{~Hz}-1 \quad|=|$

ChRger variants in cultured neurons and in acute brain slices outperform the commonly used ChR2(H134R) and the high performance CoChR. (a) ChRs were cloned into an AAV vector with either the hSyn or CamKIIa promoter and packaged into rAAV-PHP.eB for expression in culture and in vivo. (b) Cultured neurons expressing ChRgers and ChR2(H134R) under the hSyn promoter (repeated independently six times per construct with similar results). (c) Peak and steady-state photocurrent with low-intensity $\left(8 \times 10^{-3} \mathrm{~mW} \mathrm{~mm}^{-2}\right)$ and moderateintensity $\left(0.8 \mathrm{~mW} \mathrm{~mm}^{-2}\right)$ light in cultured neurons (ChR2, $n=16$ cells; CoChR, $n=17$ cells; ChRger1, $n=9$ cells; ChRger2, $n=24$ cells; ChRger3, $n=9$ cells). (d) Spike fidelity with varying intensity light for $5 \mathrm{~ms}$ light-pulse width at $2 \mathrm{~Hz}$ stimulation (ChRger1, $n=6$ cells; ChRger2, $n=6$ cells; ChRger $3, n=6$ cells; CoChR, $n=7$ cells; ChR2, $n=7$ cells). (e) Spike fidelity with varying stimulation frequency with $2 \mathrm{~ms}$ light-pulse width in cultured neurons (ChRger1, $n=9$ cells; ChRger2, $n=12$ cells; ChRger3, $n=7$ cells; ChR2, $n=8$ cells). (f) Representative voltage traces of ChRgers and ChR2(H134R) at $2 \mathrm{~Hz}$ with $5 \mathrm{~ms}$ pulsed low-intensity blue light stimulation $\left(3 \times 10^{-2} \mathrm{~mW} \mathrm{~mm}^{-2}\right)$ shows robust neuronal firing for ChRgers while ChR2(H134R) and CoChR exhibit only sub-threshold light-induced depolarization. (g) Photocurrent strength with varying light irradiances in acute brain slice after direct injection of rAAV-PHP.eB packaged hSyn-ChR constructs into the PFC (ChRger1, $n=11$ cells; ChRger2, $n=11$ cells; ChRger3, $n=11$ cells; ChR2, $n=9$ cells) or after systemic delivery of CamKIIa-ChRger2 (ChRger2, $n=6$ cells; $\left.5 \times 10^{11} \mathrm{vg} / \mathrm{animal}\right)$. (h) Representative current traces of ChRgers and ChR2(H134R) with a $300 \mathrm{~ms}$ light pulse at 
varying light irradiances in acute brain slice after direct injection. (i) Systemic delivery of rAAV-PHP.eB packaged hSyn-ChRger2 or hSyn-ChR2(H134R) resulted in broad expression throughout the cortex $\left(5 \times 10^{11} \mathrm{vg} /\right.$ animal; repeated independently five times per construct with similar results). (j) The fraction of light excitable neurons in the PFC after systemic delivery of hSyn-ChRs measured by cell-attached recording in acute slice targeting only neurons expressing the eYFP marker $\left(1 \times 10^{11} \mathrm{vg} /\right.$ animal). Peak (solid line) and steady-state (dashed line) photocurrent strength (k) and spike fidelity (l) with varying light irradiances in acute brain slice after systemic delivery $\left(1 \times 10^{11} \mathrm{vg}\right.$ /animal) of hSyn-ChRger $2(n=13$ cells $)$ and hSyn-CoChR ( $n=14$ cells) (recorded in PFC neurons). (m) Spike fidelity with varying stimulation frequency in acute brain slice after systemic delivery $\left(1 \times 10^{11} \mathrm{vg} / \mathrm{animal}\right)$

(CoChR, $n=15$ cells; ChRger1, $n=9$ cells; ChRger2, $n=5$ cells; ChRger $3, n=8$ cells) with $1 \mathrm{~mW} \mathrm{~mm}^{-2}$ intensity light. (n) Representative voltage traces with blue light-driven ( $1 \mathrm{~mW}$ $\mathrm{mm}^{-2}$ ) spiking at the indicated frequencies. vg, viral genomes. Plotted data are mean \pm SEM. 
a

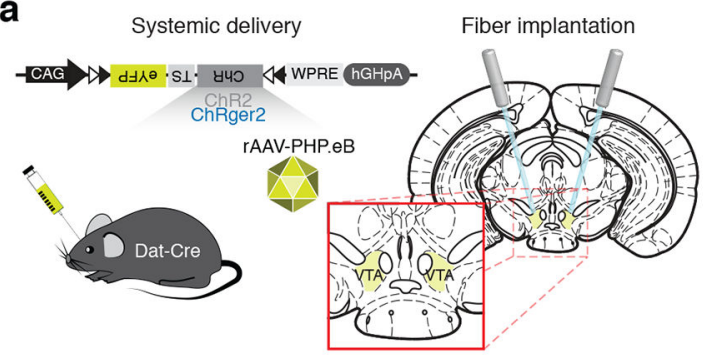

d
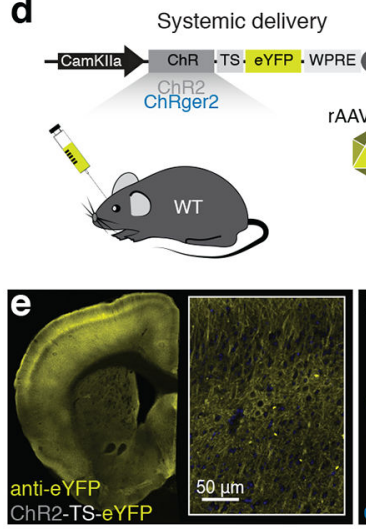

Fiber on skull surface
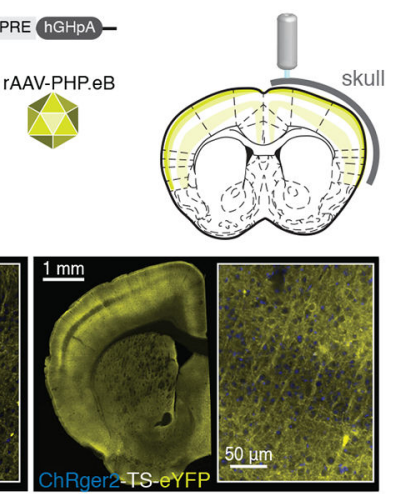

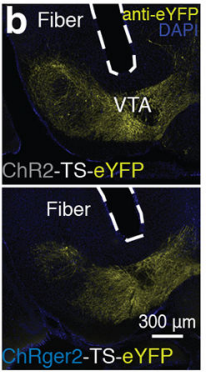

C
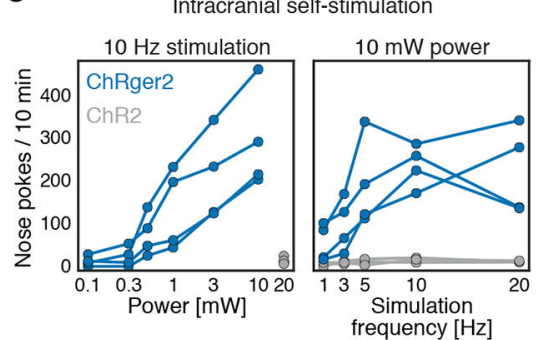

f

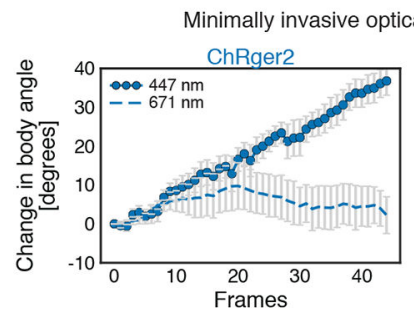

g

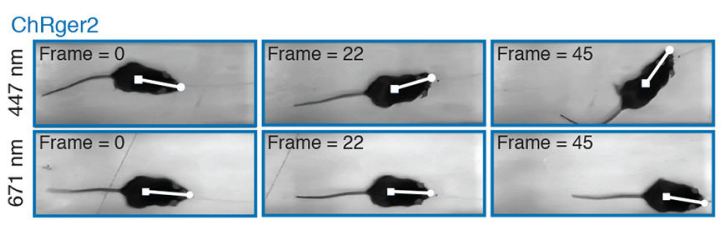

Figure 4.

Validation of high-performance ChRger2 for minimally-invasive optogenetic behavioral modulation. (a) Systemic delivery of rAAV-PHP.eB packaged CAG-DIO ChRger2-TS-eYFP or ChR2(H134R)-TS-eYFP $\left(3 \times 10^{11} \mathrm{vg} /\right.$ mouse) into Dat-Cre animals coupled with fiber optic implantation above the VTA enabled blue light-induced intracranial self-stimulation (ten 5 ms laser pulses) exclusively with ChRger2 and not ChR2(H134R) with varying light power and varying stimulation frequencies. ChRger2, $n=4$ animals; ChR2(H134R), $n=4$ animals. Images show fiber placement and opsin expression for ChR2(H134R) (top) and ChRger2 (bottom). (b) Minimally-invasive, systemic delivery of rAAV-PHP.eB packaged CaMKIIa ChRger2-TS-eYFP or ChR2(H134R)-TS-eYFP $\left(5 \times 10^{11} \mathrm{vg} / \mathrm{mouse}\right)$ into wild type (WT) animals coupled with surgically secured fiber-optic cannula guide to the surface of the skull above the right M2 that had been thinned to create a level surface for the fiber-skull interface. Three weeks later, mice were trained to walk on a linear-track treadmill at fixed velocity. Coronal slices show expression throughout cortex with higher magnification image of M2 (inset) for ChR2(H134R) (left) and ChRger2 (right). Unilateral blue light stimulation of M2 induced turning behavior exclusively with ChRger2 and not ChR2(H134R) (10 Hz stimulation with $5 \mathrm{~ms} 447 \mathrm{~nm}$ light pulses at $20 \mathrm{~mW}$ ). ChRger2, $n=5$ animals; ChR2(H134R), $n=5$ animals. No turning behavior was observed in any animal with $10 \mathrm{~Hz}$ stimulation with $5 \mathrm{~ms} 671 \mathrm{~nm}$ light pulses $(20 \mathrm{~mW})$. Plotted data are mean \pm SEM. vg, viral genomes. 